\title{
Protée
}

\section{Échographie imaginaire : formes et figures poétiques d'un impensé intrafoetal dans l'oeuvre de Michel Leiris}

\section{Sylvie Boyer}

Volume 35, numéro 1, printemps 2007

Échos et résonances

URI : https://id.erudit.org/iderudit/015891ar

DOI : https://doi.org/10.7202/015891ar

Aller au sommaire du numéro

\section{Éditeur(s)}

Département des arts et lettres - Université du Québec à Chicoutimi

ISSN

0300-3523 (imprimé)

1708-2307 (numérique)

Découvrir la revue

Citer cet article

Boyer, S. (2007). Échographie imaginaire : formes et figures poétiques d'un impensé intrafoetal dans l'oeuvre de Michel Leiris. Protée, 35(1), 75-84.

https://doi.org/10.7202/015891ar
Résumé de l'article

Être appelé à la vie au nom de la mort - au nom d'une mort(e) -, voilà qui caractérise le statut d'enfant de remplacement de Michel Leiris. On peut penser qu'au miroir de l'autobiographie, l'écrivain a tenté (tel Narcisse qui, dans l'une des versions du mythe, recherche dans la vision de son propre reflet à la surface de l'eau une soeur jumelle disparue) de rencontrer une imago sororale gémellaire, de capter les échos et résonances d'un alter ego. Toutefois, ce sont les textes poétiques de Leiris qui, peut-être mieux que n'importe quels écrits leirisiens, donnent à penser les formes et les figures d'une « mort en transmission " ou, pour reprendre les termes du poète, d'une " malemort ". L'étude de champs sémantiques relatifs à l'univers placentaire et foetal, dans l'oeuvre poétique de Leiris, permet de cerner - à l'instar d'images échographiques - la persistance d'une présence mortifère que l'écriture leirisienne s'emploie à traduire et à nommer. 


\title{
ÉCHOGRAPHIE IMAGINAIRE: FORMES ET FIGURES POÉTIQUES D'UN IMPENSÉ INTRAFOETAL DANS L'OEUVRE DE MICHEL LEIRIS
}

SYLVIE BOYER

\author{
Telle devant la niche où dort un saint de pierre \\ foetus rêvant de tout son crâne déplumé \\ et muet dans l'utérus comme un mort dans sa terre \\ coule une cire que l'ardeur de sa flamme fait suer. \\ Michel Leiris, «La Néréide de la mer Rouge» (1969: 124) \\ L'autre nuit est toujours l'autre, et celui qui l'entend devient l'autre, \\ celui qui s'en rapproche s'éloigne de soi, n'est plus celui qui s'en \\ rapproche, mais celui qui s'en détourne, qui va de-ci, de-là. Celui qui, \\ entré dans la première nuit, intrépidement cherche à aller vers son \\ intimité la plus profonde, vers l'essentiel, à un certain moment, entend \\ l'autre nuit, s'entend lui-même, entend l'écho éternellement répercuté de \\ sa propre démarche, démarche vers le silence, mais l'écho le lui renvoie \\ comme l'immensité chuchotante, vers le vide, et le vide est maintenant \\ une présence qui vient à sa rencontre.
}

Maurice Blanchot, L'Espace littéraire (1955: 222)

Michel Leiris fut un enfant de remplacement. Dans une notice placée en annexe de son Journal posthume, l'écrivain-autobiographe fait allusion au désir de sa mère, alors qu'elle était enceinte de lui, d'avoir une fille qu'elle voulait prénommer Micheline afin de remplacer une enfant morte en bas âge ${ }^{1}$. Dans la biographie qu'elle a consacrée à Michel Leiris, Aliette $\mathrm{Armel}^{2}$ précise que l'enfant décédée se prénommait Madeleine et qu'elle est morte à l'âge de quatre ans. Bien que quatre ans, justement, séparent la mort de l'enfant de la naissance de Michel Leiris et que deux frères sont nés à la suite de ce décès, tout laisse croire, comme en témoigne la notice du Journal, que c'est au petit dernier de la famille que revient la charge de porter le deuil non fait de la mère.

Dans un texte intitulé Grande Fuite de neige, Michel Leiris écrira: «Je suis comme un mort, ou plutôt comme quelqu'un qui jamais ne serait né» ${ }^{3}$. Or, les sentiments d'inexistence et de dépossession qui traversent l'œuvre de l'écrivain, de 
même que l'obsession de la mort, ainsi que la question de l'ambivalence sexuelle qui revient tel un leitmotiv, peuvent être interprétés à l'aune de cette problématique des origines dont la psychanalyse et la psychopathologie ont montré toute l'importance, tant du point de vue des effets psychiques sur le sujet venu à la vie en lieu et place d'un autre, qu'en regard de l'acte créateur ${ }^{4}$. Michel Leiris s'inscrit ainsi dans la lignée des écrivains et artistes enfants de remplacement qui ont trouvé dans la création un moyen de reconnaissance et une façon de se différencier de l'Autre. Dans cette perspective, le projet identificatoire que constitue l'entreprise autobiographique leirisienne, celui d'une démarche d'autohistorisation, prend un sens particulier. Il répond à un travail de reconstruction qui consiste à écrire et à réécrire sans cesse une histoire de vie dont le sujet-autobiographe se sent dépossédé.

On peut avancer que cette problématique des origines - celle d'une mort d'enfant qui habite et hante l'histoire familiale et la préhistoire personnelle de Michel Leiris - est à la source, chez cet écrivain, de la création. Ce serait autour de cette question restée en souffrance de représentation, laquelle, dans toute l'œuvre, fait l'objet d'un véritable blanc à l'instar du nom de la morte, Madeleine, qui jamais n'est donné, que tournerait l'écriture leirisienne. Le nom de la morte, telle une madeleine invisible, est peut-être ce frêle bruissement de l'œuvre de Leiris qui, à travers le nom de Micheline, puis à travers celui de Michel, fait résonner l'écho d'une voix spectrale, voix d'outretombe, qui appelle et rappelle le sujet de l'écriture comme autre.

Ainsi, la voix de l'écriture prend racine à même un territoire sombre et infernal, figure de l'espace interne où bée l'abîme incommensurable de la mort:

[...] dans le mot "mort", l'unique voyelle est o, dont le son se prolonge comme un frappement de cloche tintant d'un bout à l'autre d'une galerie couverte, tandis que le cercle par lequel cette voyelle est figurée bée en plein milieu du mot, comme l'entrée d'un tunnel, la bouche d'un égout ou l'orée de toute espèce de couloir souterrain qui peut se faire canal d'échos répercutés.

(BIF: 47)
De ce $o$ de la mort se répercute dans l'œuvre leirisienne l'écho d'une résonance sonore ancienne, celle des " restes vivaces ${ }^{5}$ - "vivantes cendres, innommées", telles que l'indique ce titre d'un recueil de poèmes de Haut Mal (217-230) -, ou de la «survivance " 6 d'une voix féminine qui ne cesse de se faire entendre telle une onde dans la mémoire familiale.

On l'aura compris: en tant qu'incarnation fantasmée d'un autre enfant, l'enfant de remplacement figure de façon métaphorique la personnification même de l'écho ou d'Écho condamnée à réfléchir, à refléter, à répercuter une présence d'absence. Au miroir de l'autobiographie, Narcisse n'est pas seul. Michel Leiris, écrivain enfant de remplacement, n'aura cessé à cor et à cri de cerner et de rechercher les traits d'un visage «autre» - telle une imago gémellaire ou une sœur en miroir - pour s'y reconnaitre et, en même temps, pour s'en différencier.

Ce sont les résonances de ce que j'appellerai une «mort en transmission» qu'au cours de ces pages il s'agira de traquer dans l'œuvre leirisienne. C'est à une telle "malemort» (LPC: 68), qui, peut-on penser, renvoie à cette morte que la mère n'a pas su faire mourir, que ne cesse de nous convier l'imaginaire leirisien. Telles autant d'images "échographiques», les textes poétiques de Michel Leiris (et de proses poétiques, comme Aurora), tout particulièrement, donnent à penser de même ce qui, de la nuit originelle - l'autre nuit - des territoires prénataux, intra-utérins, est antérieur au langage, mais qui s'inscrit dans le corps. De ce «trop» du trauma - et de cet autre en soi, étrange "visiteur du moi» 7 - dont la chair porte la marque, l'écrit se fait chambre d'échos.

\section{ELLE, LA MORT}

[...] m'y voici venu à la Mort cathédrale, à cette troisième singulière personne que tout à l'heure je biffais d'un trait de plume, la Mort, fourche grammaticale qui assujettit le monde et moi-même à son inéluctable syntaxe, règle qui fait que tout discours n'est qu'un piètre mirage recouvrant le néant des objets, quels que soient les mots que je prononce et quel que soit le JE que je mette en avant... (AUR: 40-41) 
«La persévérance mélancolique des os» (AUR: 60), voilà une formule saisissante qui traduit la persistance d'une présence mortifère, si ce n'est cadavérique, qui, dans toute l'œuvre de Michel Leiris, ne cesse d'insister. Tout se passe comme si le travail de l'écriture, chez cet auteur, s'employait à symboliser une identification à la vie d'outre-tombe de l'autre mort. En lisant Leiris, on pourrait croire en effet, par la représentation qui est donnée dans l'ensemble de l'œuvre d'un corps blessé, mis à mal, que ce sont les signes de décomposition d'un tel corps mort interne qui persistent en lui. Celui qui, dans L'Âge d'homme, confie («sans aucune exagération littéraire», dit-il) être «rongé» $(\mathrm{AH}: 27)$ et qui, dans son Journal, affirme se sentir, physiquement et moralement, «squelettique» (341), écrit également, dans une notice rédigée en 1936, ces propos qui laissent songeur: «Depuis un an ou deux, je ne fais plus que - cahin-caha - descendre la pente. Conesthésie du sexe mou, des entrailles relâchées. Préfiguration du blessé qui perd ses tripes, du cadavre qui se vide" (308). Salvador Dali, enfant de remplacement célèbre, a rendu compte des effets mortifères d'une telle identification: "Je me crus mort avant de me savoir en vie... Mon psychiatre préféré, Pierre Roumeguère, affirme que, identifié par force à un mort, je n'avais pas d'image véritablement sentie de mon corps autre que celle d'un cadavre putréfié, pourri, mou, rongé de vers» (1973: 297).

C'est en ce sens que, chez Leiris, il est possible d'interpréter l'idéal de roideur, de même que tout ce qui se rapporte au fantasme de minéralisation et de pétrification qui traverse l'œuvre: ils constituent un moyen de défense contre la mort vécue comme une menace constante. On connaît l'attirance du narrateur leirisien (en témoigne suffisamment L'Âge d'homme) pour l'inanimé, pour ces corps (statues de marbre, de plâtre ou encore de bronze) froids et rigides, sans viscères ni peau, qui, ainsi, échappent au morcellement, à la décomposition et à la pourriture ${ }^{8}$.

Jean-Bertrand Pontalis l'a noté, «c'est dans le discours fantasmagorique d'Aurora qu'on voit sans doute le plus clairement comment [chez Leiris] la mort et le moi sont les deux termes d'une seule obsession" (1968: 326). Le personnage d'Aurora, qui cristallise cet autre absolu, Elle, la Mort, présentifie, mieux que n'importe laquelle des figures féminines de l'œuvre de Leiris, le fantôme qui hante le sujet de l'écriture. Rivés l'un à l'autre comme un «cadavre à son linceul» (AUR: 60), c'est bien un couple - un étrange couple - que forment Aurora et Damoclès Siriel, le narrateur de ce texte qui porte le nom anagrammatique de Leiris. La relation duelle gémellaire - entre les membres de ce "couple" apparaît comme le redoublement du dédoublement qu'instaure la "couplaison" Leiris/Siriel. Aux origines de l'autobiographie, Aurora scelle ainsi «l'alliance du moi, de la mort et du double» (Pontalis, 1968: 327). Aurora, fantôme abhorré et aimé, figure du soi et de l'autre, est l'image même, dans l'œuvre leirisienne, du lien unissant l'Éros et la mort. Comme d'une seconde peau identitaire (peau de chagrin, peau d'âne ou outre de sang cousue à même la chair), c'est de ce corps spectral, ou «vêture de fantôme» (AUR: 100), qu'est paré le narrateur d'Aurora, à l'image du diadème fait du sang desséché de ses victimes que porte Siriel nuit et jour, seul dans son palais, et qui rappelle le collier de perles ornant le cou d'Aurora, ces «perles nées de ses os délicats irisés par la blancheur lunaire de sa chair disparue» (AUR: 131).

Pour «l'enfant dédoublé» qu'est l'enfant de remplacement, l'emploi d'une doublure anagrammatique, ciselant à même le signifiant la forme inversée et réfléchissante d'un «autre pareil à soi ", permet peut-être de donner corps à l'invisible double gémellaire que, depuis toujours, l'autobiographe «traîne» avec lui. Selon Andrea Sabbadini, cette psychanalyste qui a le plus insisté sur le sentiment de confusion identitaire particulier à la condition d'enfant de remplacement, ce dernier, en effet, " "traînerait” toujours avec lui comme un double ou un jumeau l'enfant mort, ce double devenant à la fois le dépositaire des idéalisations, mais aussi des désirs de meurtre» (1989: 519). En écho à ces propos, lisons encore Salvador Dali: «Van Gogh est devenu fou de la présence d'un double mort à ses côtés. Pas moi. [...] Je suis né double avec un frère en trop, qu'il 
m'a fallu d'abord tuer pour acquérir ma propre place, mon propre droit à ma propre mort» (1973: 16). Or, si l'invention d'un double anagrammatique - Siriel/ Leiris, qui reflète peut-être cette autre couplaison, Michel/Micheline, laquelle constituerait à mon sens la signature secrète, duelle, originelle de l'œuvre leirisienne - a permis à Leiris d'opérer un processus de séparation, de distanciation consistant à se voir "hors-là» afin de se protéger de la mort et de se dégager d'une identification mortifère, on peut avancer que le personnage d'Aurora, qui est la création d'un corps écrit sans cesse démembré, décomposé et reconstitué, autorise, sur la scène littéraire, le meurtre symbolique, toujours à recommencer, de l'enfant morte.

\section{PARLER LA LANGUE DU CORPS}

Encore moins de mots. Mes idées ressemblent de plus en plus à de simples sensations organiques.

(Leiris, Journal 1922-1989, 1992: 100)

J'ai maintes fois évoqué l'existence et la mort de ce frère aîné, dont j'ai trouvé les traces dès que mon attention s'est éveillée vêtements, portraits, jouets - et qui avait laissé dans la mémoire de mes parents des souvenirs affectifs indélébiles. J'ai ressenti profondément la persistance de cette présence à la fois comme un traumatisme - une sorte de vol d'affection - et une exaltation de dépassement. [...] Le désespoir de mes parents ne fut calmé que par ma propre naissance, mais leur malheur imprégnait toutes les cellules de leur corps. Dans le ventre de ma mère, je ressentais déjà leur angoisse. Mon fotus baignait dans un placenta infernal. Cette angoisse ne m'a jamais quitté.

(Dali, 1973: 12-13)

Les textes poétiques de Michel Leiris, qui touchent un «irrationnel interne, tramé dans la viscéralité où se nouent les pulsions d'Éros et de mort" (Sermet, 1997: 179), pointent une zone prélangagière, quelque chose d'avant le symbolique (quelque chose de la naissance, de la mort et d'un "cadavre outre-signifiant", pour reprendre l'expression de Jean-Thierry Maertens [1979]) qui n'advient pas au discours. La lecture de recueils de poèmes comme Simulacre et La Néréide de la mer Rouge, celle des récits de proses poétiques tels Le Point cardinal, Aurora ou encore celle des matériaux inédits de l'époque surréaliste («Les foraminifères» et "L'évasion souterraine») publiés après la mort de Leiris et réunis, établis et présentés par Catherine Maubon sous le titre L'Évasion souterraine, constitue une véritable plongée dans des territoires intérieurs sombres et étrangement inquiétants, hantés par la mort et ses fantômes. Il convient de rappeler que, de façon générale, Michel Leiris rejetait ce pan de son écriture resté en marge de l'œuvre. C'est le cas des fragments de L'Évasion souterraine, demeurés volontairement inédits tout au long de sa vie et maintenus dans un «état de prématuration». Ces œuvres «fœetales», dirais-je, non abouties, constituent - en tant que «lieu d'expérimentation du difficile passage des images mentales aux mots qui auraient dû leur correspondre, de la représentation de soi à la narration de soi» (Sermet, 1997: 14) - les racines à partir desquelles s'érigera l'œuvre future. La langue poétique est peut-être la seule forme qui permette de traduire au plus près une «malemort».

Il est entendu que «le monde de l'origine ne s'observe pas: il se pense et se réfléchit, il se médite à partir de quelques données fondamentales et de leurs effets insoupçonnés » ${ }^{9}$. En cernant l'existence de champs sémantiques qui font référence à l'univers intrafoetal, il s'agit ici de faire partager au lecteur, par cette "échographie imaginaire», le sentiment, à la lecture de l'œuvre poétique de Michel Leiris, de pénétrer une part de la sphère archaïque mère-enfant et d'apercevoir les ombres qui y circulent, et ce, en acceptant de laisser à l'inconnu sa part d'énigme. C'est la persistance d'une présence vécue comme traumatisme, pour reprendre les termes de Dali, que nous retrouverons. Dans la bulle précoce mère-enfant suivant en cela la notion théorique de sphère avancée par Peter Sloterdijk pour penser en termes de forme et d'espace la relation «bi-unitaire» qui se noue dans la bulle entre l'enfant et la mère ${ }^{10}$-, coexistent en une trop grande intimité, chez Leiris, un mort et un vivant.

Parler la langue du corps, parler la langue de sensations organiques originaires, parler «la langue des 
radiations cellulaires» (HM: 47) - celle des «faisceaux médullaires» (HM: 23), des «trottoirs lymphatiques» (HM: 112), des «lacis de viscères» (HM: 187), des «tourbillons moléculaires» (LPC: 34-35), des "circonvolutions dans les ténèbres prismatiques" (LPC: 45) -, tel est l'effort d'une part du discours poétique leirisien pour entrer en possession de ce qui, forcément, est de l'ordre d'une outre-signifiance:

[...] je voudrais toujours descendre

pareil au fer d'une bêche qui coupe dans la terre le sillon rectangulaire des tombes

descendre dans cette nuit plus bas que les tropiques souterrains (HM: 57 )

Descendons avec le poète à l'intérieur du corps matriciel exposé notamment dans Aurora, dans la «chambre souterraine carrée [de] la matrice» (AUR: 91), de même que dans l'enceinte "d'Aigues-Mortes" qui en est un avatar - cette ville plongée depuis longtemps «dans une nuit de café noir» (AUR: 178) pour tenter de cerner les formes et les figures d'une présence d'absence.

Dans la «nuit utérine», une «nuit plus noire que le sang» (HM: 51), à travers les échanges sanguins mèreenfant, quelque chose d'un «fluide mortel» (SIM: 16) se propage qui est vécu comme effraction. Donneuse de vie, la mère est surtout dans l'œuvre de Leiris donneuse de mort. Le poème La Néréide de la mer Rouge, par exemple, en témoigne suffisamment: «[...] son ventre chargé de futurs ossements fait de la femme pleine un sépulcre mouvant» (HM: 125). Si l'équivalence entre le berceau originel et le tombeau est incontournable, il appert que l'association constante que l'on retrouve, dans les textes de Leiris, entre les entrailles maternelles et la mort dépasse cette idée d' "enténèbrement" d'une sorte de mort prénatale et qu'elle va au-delà, de même, de l'évident et irréversible constat du don de vie comme ultime don de mort. Bien plus, la mort apparaît chez Leiris comme un poison qui, dès avant la naissance - dès la vie prénatale, donc -, et son existence durant, ronge les chairs et brûle l'âme. C'est de cette causticité que tente de se libérer l'écrivain en transférant au corps de l'œuvre - dans un geste à la fois nécessaire et illusoire - son venin au moyen d'un crayon (flèche d'Héraclès ou épée de Damoclès) trempé au sang de l'hydre:

Tant qu'à la froide neutralité de la feuille blanche en divaguant

je n'aurai pas infusé sa brûlure,

le poison qui me tourmente

ne cessera de ronger mes entrailles. (HM: 243)

La mère, la "mer Rouge", par homonymie, exhale son venin et «ronge comme un acide» les pays lointains qui, depuis toujours, hantent le voyageur. N'est-ce pas elle, la mère, éclaboussée de sang, qui, un jour, mit en terre son enfant? Les images maintes fois réitérées d'entrailles maternelles "détestables» (HM: 68) - le "vagin des meurtrières" (LPC: 63) -, sanglantes, conteneurs d'ossements, de «tiges funestes» (HM: 68), et "empuanties par l'herbe de tristesse" (AUR: 112) évoquent l'intérieur d'un corps qui fait figure de tunique empoisonnée. Est-il possible d'imaginer un fœetus nageant dans «l'écume atroce d'une mer de tourments" (ES: 114), à travers un "fleuve d'ossements» (ES: 113) et en proie au «fracas terrible des rencontres artérielles» (ES: 114)? Ne serait-ce pas, avant même le don de vie, ce don de mort qu'en gage d'amour et de fidélité à l'enfant mort la mère offre à l'enfant substitut? Ne voit-elle pas qu'à l'aube des sens ce présent funeste le brûle déjà? La lecture de l'œuvre de Michel Leiris révèle que les "pigments de la mort» (ES: 90) peuvent s'incruster dans la peau, telle une marque de naissance imperceptible que porterait le corps, sorte de nævus ou d'«envie» qui, selon la croyance populaire, constitue la trace d'une envie de la mère. Ce secret de la mort, secret de la mère, secret d'une naissance intriquée à une mort, s'inscrit, aux origines, dans la chair qui tout à la fois le porte et l'innomme.

Le passage suivant, tiré d'un court texte appelé "Les foraminifères - Traité des maladies mortuaires", me semble illustrer de façon tout à fait exemplaire la forme que peut revêtir, dans l'imaginaire, l'invasion contaminante et mortifère d'un imperceptible corps étranger : 
Les foraminifères, êtres microscopiques, vivant dans des coquilles percées de trous qu'ils abandonnent en colonies de pierre.

Somptueuses maladies de la mort, vous les connaissez bien.

Des millions d'êtres. Hors des circuits.

Carnassiers, je broierai tous vos membres. L'illicite comédie du temps, enfin, va déchaîner les pierres. Une grêle de flèches insaisissables. Les replis du cerveau.

À travers les vêtements de pierre dure, les foraminifères... [...]

Le rythme des pensées veules veut une circulation de larmes, qui remplacerait, en poésie, la circulation du sang?

[...] et tu surgis, enfant voleur armé des migrations, en proie aux colonies de bêtes cachées, crissement de bagarres vagabondes, ô Maladie!

Maladie? Mortuaire plus que mortelle, en raison de la lueur, du suaire plus stable que la selle. Migration folle, indéfinie, hors du château absurde des continents, métamorphose, bloc foré par des milliers de dards... (LF: 68-69)

Les foraminifères n'incarnent-ils pas en effet la figure d'un «autre-que-soi» interne qui vit en parasite et qui, indésirable et redoutable invité, intoxique le corpspsyché de l'hôte à l'intérieur duquel il loge? Il y aurait peut-être lieu, en ce sens, de parler d'une sorte de vampirisme ou alors de cannibalisme foetal ${ }^{11}$ d'ordre psychique. Dans les textes et les récits poétiques de Michel Leiris, le sujet de l'écriture est constamment en lutte avec une ombre - «algues carnassières» (ES: 71), «aigue marine» (HM: 45) - qui l'emprisonne, l'étouffe et menace de l'avaler. C'est une contamination des territoires psychiques du soi et de l'autre, c'est cette étrange cohabitation des morts avec les vivants dans un monde de sang et d'eau que nous retrouvons dans ce dialogue qu'entend le narrateur du Point cardinal:

- Plus haut que le cadastre blanc des lunaisons, avec quoi les morts marquent-ils les limites de leur antre?

- Ils marquent leur séjour angulaire avec la cendre et les tessons du sang.

- Et toi, qu'inscris-tu dans ton domaine?

- Magique zèbre de feu, voici que le couteau devient trigone. Il s'échappe et raye indéfiniment l'aurore, les moustiques et les écharpes froissées du marécage doré. Brusque tourmente trempée du sel et de la mer, chevelure des sueurs, ô søur vorace...

(LPC: 60)
Une telle sphère archaïque, à l'intérieur de laquelle on suppose qu'il peut y avoir coexistence d'un mort et d'un vivant, apparaît comme une bulle jumelle - ou cloche de chair - que le sujet enfant de remplacement, toute sa vie, en un même mouvement, cherchera à remplacer et tentera de crever parce qu'elle est la figure d'une appartenance intime en même temps qu'objet d'aliénation:

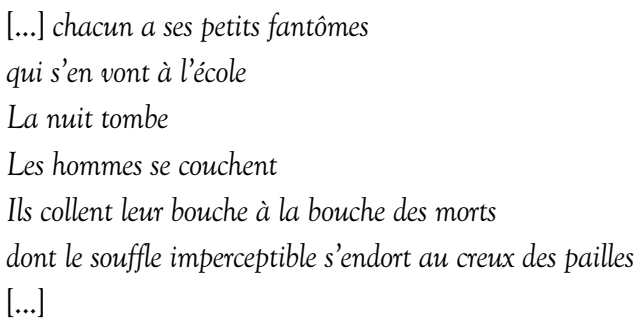

Cette étreinte d'une ombre glacée, ce collage mortifère, dit ce qu'il en est de la «résistance» des morts et de la survivance des corps. L'image de l'homme "pris» à un double mort, tel un canotier qu'on dit amarré à un corps-mort, rappelle ce personnage de Maupassant qui, sans le savoir, est fait prisonnier d'un cadavre auquel s'est accrochée l'ancre de son canot. Longtemps, c'est en vain qu'il tentera de tirer sur la chaine sans que l'ancre ne cède. Il n'osera non plus nager jusqu'à la berge puisque, dans son effroi, il lui semble qu'il se sentirait «tiré par les pieds tout au fond de cette eau noire» (Maupassant, 1973: 93).

La question du double réapparaît là dans ce lien sensible - filament, ficelle, minuscule fibrille, invisible veinule ou encore ruban de cou d'Olympia - qui attache le sujet de l'écriture à cette "ombre femelle» 12 , figure de l'Autre interne qui l'habite, étrange lymphe qui circule dans ses veines, monstrueuse nymphe ou néréide terrée, enterrée, dans le ventre de la mer Rouge:

Car au centre de la mer Rouge

couche une femme au ventre avide

aux yeux perdus signaux qui bougent

pendus à sa face livide

Ses cheveux sont une fumée 


\author{
sa bouche suceuse est exsangue \\ son cou est à jamais coupé \\ mais ses deux bras sont une cangue \\ [...] \\ Au fin fond de la mer veillait les dents lucides \\ et sa gorge fanée goudronnée de sanglots \\ guettant les suppliciés la vieille néréide \\ qu'on appelle l'Amante-aux-reflets-de-couteau \\ mais que je nomme moi maudissant mes mains vides \\ femelle de mon ombre et foudroyant pavot \\ puisque je dormirai en elle jusqu'aux ides \\ $d u$ mois vague où la terre ouvre grands ses caveaux \\ [...] \\ Les vents ont décoché pour moi l'ardente flèche \\ de l'avenir gravé d'espérance et de mots \\ mais je suis prisonnier de cette ombre que lèche \\ la gorgone qui n'a que les os sous la peau \\ Je l'appelle Ma mort Menottes d'or luisantes \\ Cave d'alcool trop fort Mère pas assez tendre \\ Lichen poussant sur les décombres qui me hantent \\ Reflet profond des yeux dont des pleurs vont descendre [...].
}

(HM: 135-136)

Tel Ulysse appelé par le chant des sirènes, Michel Leiris ne fut-il pas toute sa vie interpellé par les pleurs de la mère (gorgone léchant interminablement sa blessure), happé par une plainte funèbre contre laquelle il ne cessa de lutter pour se garder de la séduction mortelle qu'elle exerça? N'est-ce pas à la fois l'enchantement et l'effrayante démesure de cette voix - écho de l'autre et de soi - qui saisirent l'ethnologuevoyageur parti à la rencontre de lui-même lorsqu'en cette mythique Afrique il navigua sur les eaux de la mer Rouge? Résister à cor et à cri à la violence de la voix maternelle qui, à l'origine, appelle l'enfant de remplacement comme autre, voilà peut-être obscurément ce dont il s'agit. Il me semble pouvoir parler, à propos de cet étrange "chant de bienvenue» qui accueille celui qui est appelé à la vie au nom de la mort - tel un singulier éloge funèbre -, d'une sorte de viol musical primitif qui remonterait à la première alliance «sonosphérique» mère-fœetus. Le passage suivant, tiré du Point cardinal - ce récit onirique dans lequel le narrateur, parti sur les traces d'une jeune fille nommée l'Ingénue, explore, à l'instar du narrateur d'Aurora, une ville faisant figure de corps -, donne à voir et à entendre, dans ce monde de sang et d'eau, encore une fois, l'imagerie d'une présence sonore intrusive, celle d'une voix menaçante:

Battus par les vagues végétales de cette voix onduleuse, les murs imaginaires commençaient à apparaître par instants et l'on voyait leur trame comme le réseau des veines lorsque passe le sang. [...] Niveau mobile des flottaisons, la voix se boursouflait de cataclysmes maritimes et volcaniques, cyclones et laves des perceptions, qui marquaient l'air de longues traces sulfureuses [...]. La sphère écarlate de l'amour glissait le long de l'axe impondérable de la voix, tous les phantasmes déployés dans les failles profondes de cette armure dont les articulations laissaient transparaître la pensée. Voix sanglante, essentielle, ce fut d'abord un vol de messages qui passèrent à ma gauche et s'enfuirent vers l'horizon sans désirs, avec de grandes clameurs d'oiseaux de nuit [...]. ${ }^{13}$ (LPC: 57-58)

Dans le passage cité, de même que dans ces expressions de Leiris sur lesquelles ma lecture s'est arrêtée - "arêtes sonores qui transmettent les remous" (HM: 188); «muscle sonore» (HM: 184); «leurs artères chantaient la ronde du sang» (HM: 48); «le suaire des voix» (SIM: 15) -, il m'a semblé qu'était bien donnée à entendre la résonance d'une voix maternelle originaire dont les modulations (sons viscéraux perçus comme du morse) resteraient imprégnées et «enregistrées» dans le corps. Ces images poétiques de Leiris trouvent en effet un écho saisissant dans les propos de l'oto-rhino-laryngologiste et psycholinguiste français Alfred Tomatis sur les traces d'imprégnabilité sonore du corps foetal, sur une "mémoire du corps", sur le rôle fondamental de la peau dans la perception sonique du foetus et sur les réponses musculaires de ce dernier aux sons qui l'environnent ${ }^{14}$. À travers les bruits cellulaires et moléculaires, à travers le fracas de la circulation sanguine, le bruit de digestion de la mère et le vacarme des battements de cœur, parvient au fotus la modulation première et essentielle de la voix de la mère: 
Tandis que tous les bruits qui traduisent la vie neuro-végétative de la mère se propageront au travers des tissus, se heurteront à la paroi utérine en agissant au travers du liquide amniotique, la voix de la mère selon toute vraisemblance pourra atteindre la cavité utérine et l'oreille du foetus par un cheminement le long de la colonne vertébrale de la mère, donc par transmission osseuse, laquelle jouera le rôle de filtre. Au-delà de la vie végétative de sa mère, le foetus percevra donc dans le lointain cette voix qui amorce le dialogue, qui passe le message affectif.

(Tomatis, 1981: 152)

Si le sang est l'agent premier de la transmission dans le monde intra-utérin, on constate ainsi que la voix maternelle, par la qualité de résonance du squelette de la mère (et grâce à la réceptivité foetale, notamment de l'ouie), en constitue un canal tout aussi spécifique. Le corps même de la mère, ainsi, se fait voix. Bien plus, pour reprendre l'interprétation de Tomatis, il est comme un instrument de musique capable de faire vibrer le corps fotal et de lui transmettre les plus fines oscillations de sa voix. C'est également de cette imagerie sensorielle que s'inspire Peter Sloterdijk dans de très belles pages sur la première alliance sonosphérique:

Celui-ci [l'enfant] écoute attentivement contre le bas du bassin

et la colonne vertébrale de la mère, comme un visiteur curieux

colle l'oreille à une porte derrière laquelle il suppose l'existence de secrets gratifiants. (2002: 555)

"Le stade des sirènes", comme le nomme Sloterdijk en faisant référence à Homère, évoque pour lui cette période au cours de laquelle l'enfant prête l'oreille en acceptant ou alors en refusant d'être atteint par son message - à l'appel de la mère. Ne serait-ce pas jusque dans ce lieu prénatal, là où s'amorce la faculté d'écoute de l'autre et de soi - écho d'une confuse mais ontologique perception -, dans cette co-audition fondatrice, que Leiris, fasciné par le chant, le cri et la voix (dont on retrouve la prédominance dans ses derniers écrits), recherche le frêle bruissement d'une voix intime modelée à la fois par l'enchantement et la violence de cette langue maternelle originaire et immémoriale?

\section{N O TES}

1. Le seul texte dans lequel Michel Leiris fait allusion aux circonstances qui ont entouré sa naissance - à ce statut d'enfant de substitution - est resté manuscrit. Il a été publié par l'exécuteur testamentaire de son œuvre, Jean Jamin, dans cette notice du Journal que Leiris a voulu posthume. On peut y lire: "Tout ce que je sais d'avant ma naissance, c'est que ma mère désirait une fille, venant de perdre une enfant dont je n'ai vu que des portraits; elle projetait de l'appeler Micheline» (Leiris, Journal 1922-1989, 1992: 876-877).

2. Dans cette biographie dont le premier chapitre s'intitule «Dans le secret des origines ", Aliette Armel insiste sur l'importance du statut d'enfant de remplacement qu'occupe Michel Leiris: «Malgré toute la tendresse qui l'entoure, malgré tout ce qui, au quotidien, lui apporte la preuve qu'il est un enfant chéri et désiré, Michel Leiris porte dès sa naissance un double fardeau : celui d'être l'enfant de substitution de Madeleine, la sœur morte dont l'absence mine toujours sa mère, et celui d'être né à la place de la fille attendue. Sa naissance est ainsi placée sous le signe de la mort et du trouble de l'identité " (Armel, 1997 : 30).

3. Leiris, 1982 : 15. Les prochaines références aux œuvres de Michel Leiris citées seront mises entre parenthèses dans le corps du texte selon la liste des abréviations suivantes: (AH) L'Âge d'homme; (AUR) Aurora; (BIF) Biffures; (ES) L'Évasion souterraine; (FOUR) Fourbis; (HM) Haut Mal, suivi de Autres lancers; (JOUR) Journal 1922-1989; (LF) «Les foraminifères ", dans L'Évasion souterraine; (LPC) Le Point cardinal (1927), dans Mots sans mémoire; (SIM) «Simulacre» (1925), dans Mots sans mémoire.

4. L'expression "enfant de remplacement" a été introduite par Orlow Poznanski en 1972 dans un article intitulé "The replacement child. A saga of unresolved parental grief ". Des études consacrées à cette problématique, il apparaît que l'enfant de remplacement est conçu pour prendre la place du mort et qu'il a pour fonction de faire l'économie du travail de deuil du parent. Outre l'atmosphère de deuil non liquidé dans laquelle naît le remplaçant, les chercheurs qui se sont intéressés à cette question soulignent l'importance de l'identification de l'enfant au disparu et, aussi, du sentiment de culpabilité de ce «survivant». Cette problématique s'inscrit de façon plus générale dans le cadre des recherches sur les phénomènes intergénérationnels, sur les questions de deuil, de transmission, d'encryptement et de fantôme telles que les ont théorisées M. Torok et N. Abraham (1996). Le fantôme résulte des effets sur l'inconscient d'un sujet de la crypte d'un autre, c'est-à-dire, dans le cas qui nous intéresse, d'un deuil secret, indicible, parce que non fait. Ce n'est pas la mort de l'enfant qui est indicible, innommable, mais plutôt l'investissement de substitution de l'enfant subséquent, cet "enfantôme " pour paraphraser le roman de Réjean Ducharme. Ajoutons que le statut de «remplaçant» ne peut être attribué d'avance à l'enfant qui naît subséquemment à un enfant mort. Il importe ainsi de souligner la part d'appropriation fantasmatique du sujet qui se vit comme remplaçant un autre, et ce, pour acquérir la place la plus investie auprès de la mère. Sur ces questions, nous renvoyons le lecteur aux articles de M. Hanus (1982), H. Urban (1989), H. Brunetière (1990), S. Consoli (1983). Par ailleurs, Maurice Porot a retracé plusieurs figures d'écrivains et d'artistes enfants de remplacement - dont François-René de Chateaubriand, Stendhal, Vincent Van Gogh, Ludwig Van Beethoven, Camille Claudel, Marie Cardinal, Louis Althusser, Salvador Dali, etc. - et s'est intéressé, dans une perspective psychopathologique toutefois, aux diverses traces de cette question des origines retrouvées dans leurs œuvres (1994).

5. Expression utilisée par Evelyn Granjon lors du 2e Congrès 
international de thérapie familiale psychanalytique ( La part des ancêtres. Le transgénérationnel dans les thérapies psychanalytiques du couple et de la famille») tenu à Montréal du 3 au 6 août 2006. 6. Cette formule est empruntée à l'ouvrage de Janine Altounian (2000) qui en porte le titre et qui montre l'importance du facteur traumatique (collectif, familial, générationnel) dans l'écriture de soi. La survivance est une présence d'absence que l'écriture ne peut que reconstruire.

7. Cette expression est empruntée à Alain de Mijolla (1981).

8. Ce fantasme de conservation du corps qui habite l'œuvre leirisienne (le corps écrit leirisien se veut d'ailleurs la projection imaginaire d'un corps auto-engendré, embaumé, immortalisé) trouve une résonance actuelle dans les domaines de l'art et de la science. Pensons notamment à l'exposition «Art Anatomique » du médecin Gunther Von Hagens, dans laquelle sont mis en scène de véritables corps humains (donnés à la science) conservés intacts grâce à un procédé de plastination qu'il a lui-même mis au point et qui serait comparable à la momification ou à l'embaumement des corps. Cette méthode consiste à « retirer sous vide l'eau et la graisse des tissus et de les remplacer par du caoutchouc au silicone ou de la résine époxy. Les corps entiers ou les organes gardent ainsi leur plasticité, sont inodores et se conservent pour l'éternité. [...] Débarrassé de sa peau, le corps est littéralement "écorché mort” ". En ligne: http://www.artezia.net/artgraphisme/art/vonhagens/vonhagens.htm (page consultée le 17 janvier 2007). Ces dépouilles rappellent étrangement les sculptures de Giacometti qui fascinèrent tant Michel Leiris. Par ailleurs, la question de l'enfant de remplacement elle-même fait écho à l'imaginaire clonal qui, sur une autre scène, hante notre culture. Je pense ici à tout ce qui relève de la reproductibilité technique, mais aussi aux biotechnologies de reproduction. En est une bonne illustration le témoignage de parents, qui, lors d'un documentaire télévisuel, affirmaient avoir prélevé des cellules de la peau de leur enfant morte dans l'espoir qu'un jour la mère puisse en porter le clone: «notre enfant va pouvoir revivre ", affirmait-elle à l'émission Découverte (Société Radio-Canada, 12 janvier 2003. En ligne: http://www.radio-canada.ca/actualite/ Decouverte/dossiers/70_scienceConscience/clonage_06a.html). 9. Voir l'avant-propos de J.-M. Delassus (2001).

10. «Le premier volume de cette trilogie des sphères parle d'entités microsphériques qui porteront ici le nom de bulles. [...]. Notre analyse s'attaque au projet, encore jamais entrepris, de raconter pour des intelligences adultes l'épopée des bi-unités toujours perdues et pourtant jamais totalement effacées. Nous plongeons dans une histoire disparue qui témoigne de la floraison et du naufrage de l'Atlantide intime; nous explorons un continent insufflé dans la mer matriarcale que nous avons habitée dans une époque subjectivement préhistorique, et abandonnée avec le début de ce qui avait l'apparence d'histoires personnelles. [...] Si le terme de pénétration était le mot juste, nous pourrions dire que nous pénétrons dans le royaume des fantômes intimes" (Sloterdijk, 2002: 70-71).

11. On retrouve ce motif du cannibalisme foetal dans la pièce de théâtre Forêts de Wajdi Mouawad. L'une des protagonistes de la pièce, qui souffre d'un cancer, apprend par ses médecins qu'un corps étranger fait partie intégrante de son cerveau. Il s'agit d'un os qui appartient non pas à lui, en propre, mais à un jumeau qui n'aurait jamais vu le jour. Dans cette pièce qui s'intéresse à la transmission transgénérationnelle à travers six générations, un tel corps étranger interne apparaît comme la métaphore de ce dont le sujet, quant à sa préhistoire personnelle, est porteur sans le savoir.

12. Le texte de Nabokov, «Scène de la vie d'un monstre double" par exemple, qui met en scène un couple de frères siamois reliés à hauteur du cordon ombilical par une bande charnue de cartilage, a l'heur de présentifier dans la matérialité des corps cette sorte d'emprise gémellaire. Il en est de même du recueil de nouvelles On pense si peu à l'amour (Actes Sud), de la Torontoise Barbara Gowdy, dans lequel on retrouve notamment le personnage d'un homme affublé depuis sa naissance d'une seconde tête malfaisante (anomalie qui « est aussi une présence familière, constitutive de l'identité») et celui d'une femme sous les jupes de laquelle se cache "une troisième jambe et un second bas-ventre appartenant à Sue, ainsi nommée par sa mère, qui en a fait une sœur siamoise inaboutie». Voir à ce sujet l'article de M.-A. Lamontagne (2004 : 29).

13. Cette présence sonore intrusive rappelle un souvenir d'enfance de Michel Leiris rapporté dans le chapitre "Mors" de Fourbis, souvenir traumatisant lié à un bruit grêle, d'origine inconnue, qui (lors d'une promenade avec les parents "à l'heure d'entre chien et loup") fait irruption dans le silence de la nuit et suscite l'inquiétude de l'enfant. Ce qui se joue dans cette scène d'enfance concerne le spectre d'une altérité au sein du familier. Cette chose inconnue et étrangement inquiétante qui se manifeste à travers l'unicité de ce bruit provenant de l'extérieur fait entendre le murmure de «la chose sans nom qui est en nous et dont le flux nous menace" (FOUR: 26). Et cette chose sans nom qui menace l'intégrité narcissique n'est pas autre chose, justement, qu'Elle, la Mort, cette inconnue. "Mors", voilà la marque profonde, mortifère morsure imprégnée dans le corps de celui qui, dans la nuit, entend la voix d'outre-tombe, écho éternellement répercuté, de l'autre, de l'autre nuit.

14. Dans ses recherches sur l'ouïe fotale, Alfred Tomatis (1981) soutient (en témoigneraient les diverses approches anatomophysiologiques) l'existence d'une perception auditive foetale dès le quatrième mois de la vie intra-utérine. 


\section{RÉfÉREN CES BIBLIO G RAPH IQ U ES}

Altounian, J. [2000]: La Survivance. Traduire le trauma collectif, Paris, Dunod.

ARMEL, A. [1997]: Michel Leiris, Paris, Fayard.

BlanCHOt, M. [1955] : L'Espace littéraire, Paris, Gallimard, coll. «Folio essais".

BRUNETIÈRE, H. [1990]: «Leurre de naissance. L'enfant de

remplacement ", Informations psychiatriques, vol. 66, n 1, 39-42.

CONSOLI, S. [1983]: "L'enfant-cicatrice», Revue freudienne, vol. 13

no 32, décembre, 21-41.

DALI, S. [1973]: Comment on devient Dali, Paris, Robert Laffont.

Delassus, J.-M. [2001] : Le Génie du fœtus. Vie prénatale et origine de

l'homme, Paris, Dunod, coll. «Enfances".

HANUS, M. [1982]: «Objet de remplacement. Enfant de

remplacement ", Revue française de psychanalyse, nº 6, 1133-1147.

LAMONTAGNE, M.-A. [2004]: "Le grand frisson des petites gens",

Spirale, dossier "Les variables de l'amour", n 198, septembre-octobre, 28-29.

LEIRIS, M. [(1939) 1993]: L'Âge d'homme, Paris, Gallimard, coll. "Folio";

[(1943) 1969]: Haut Mal, suivi de Autres Lancers, Paris,

Gallimard, coll. «Poésie/ Gallimard »;

[(1946) 1986]: Aurora, Paris, Gallimard, coll. "L'Imaginaire»;

[(1948) 1994]: Biffures, Paris, Gallimard, coll. "L'Imaginaire»;

[(1955) 1991]: Fourbis, Paris, Gallimard, coll. "L'Imaginaire»;

[(1964) 1998]: Grande Fuite de neige, Paris, Fata Morgana;

[(1969) 1989]: Mots sans mémoire, Paris, Gallimard;
[1992]: Journal 1922-1989, Paris, Gallimard, coll. "Blanche»;

[1992]: L'Évasion souterraine, textes 1924-1928 établis et présentés par C. Maubon, Montpellier, Paris, Fata Morgana.

MAERTENS, J.-T. [1979]: Ritologiques 5. Le Jeu du mort. Essai

d'anthropologie des inscriptions du cadavre, Paris, Aubier Montaigne.

MaupassanT, G. de [(1876) 1973]: "Sur l'eau", Contes fantastiques complets, Paris, Marabout, 89-95.

Mijolla, A. de [(1981) 1986]: Les Visiteurs du moi. Fantasmes

d'identification, Paris, Les Belles Lettres.

PONTALIS, J.-B. [1968] : "Michel Leiris ou la psychanalyse sans fin ", Après Freud, Paris, Gallimard, coll. «Idées », 313-335.

POROT, M. [1994]: L'Enfant de remplacement, Montréal/Paris, Éd.

Sciences et Culture/Frison-Roche.

POZNANSKI, O. [1972] : "The replacement child a saga of unresolved parental grief ", Journal of Pediatrics, vol. 81, nº 6, 1190-1193.

SABBADINI, A. [1989]: "L'enfant de remplacement », La Psychiatrie de l'enfant, vol. 37, $\mathrm{n}^{\circ}$ 2, 519-541.

SERMET, J. de [1997]: Michel Leiris, poète surréaliste, Paris, PUF, coll. "PUF écrivains".

SlOterdij, P. [(1998) 2002]: Bulles. Sphères 1, Paris, Pauvert, coll.

"Philosophie".

TOMATIS, A. [1981] : La Nuit utérine, Paris, Stock.

TOROK, M. et N. Abraham [1996] : L'Écorce et le Noyau, Paris,

Flammarion.

URBAN, H. [1989]: "L'enfant de remplacement ", Neuropsychiatrie de l'Enfance, vol. 37, no 10-11, 477-484. 